\title{
15.
}

\section{Allgemeine Bemerkungen über Rechenmaschinen, und Prospectus eines neu erfundenen Rechen-Instruments.}

(Von Herrn Ch. Z. Slonimsliy aus Bialystock in Rufsland.)

Jedem, der öfters grofse, zusammenhängende und mehrere Tage erfordernde Rechnungen auszuführen gehabt hat, ist bekannt, wie sehr anhaltendes Rechnen ermüdet und abstumpft; so dafs man öfter fehlt. : in bisweilen den Faden der Arbeit verliert und viele Umwege machen mufs. Besonders ist dies der Fall bei der Multiplication und Division vieler und so grofser Zahlen, dafs Logarithmen für dieselben nicht ausreichen. Die Producte der einzelnen Ziffern lassen sich zwar leicht aus dem Gedächtnisse finden, aber das im Sinnbehalten und Addiren der nicht hingeschriebenen Ziffern erfordert eine ununterbrochene genaue Aufmerksamkeit, und stets eine gewisse Anstrengung, welche, anhaltend fortgesetzt, sehr beschwerlich und abstumpfend ist. Die Möglichkeit von Fehlern, die bei der geringsten Unterbrechung der Aufmerksamkeit unterlaufen können, macht aber die ganze Rechnung mehr oder weniger unsicher. Noch mehr gilt Alles dies von der Division, indem man bei grofsen Divisionen oft erst versuchsweise verfahren mufs, ehe man den wahren Quotienten findet; so dafs bei so fortgesetzter ermüdender Arbeit leicht jedes bereits gefundene Resultat unsicher wird. Und wie peinlich ist es dann, die Rechnung Satz um Satz nochmals durchzusehen, um sich von der Richtigkeit des Resultats zu überzeugen.

Dieseǹ Schwierigkeiten zu begegnen, haben schon früher Männer von grofser Gelehrsamkeit, selbst Leibnitz, Mühe und Kosten aufgewendet, um ein Werkzeug zu erfinden, mittels dessen man Rechnungsresultate erhalte, die immer fehlerfrei seien und welche die beim gewöhnlichen Rechnen erforderliche Aufmerksamkeit und das Kopfrechnen ganz oder zum Theil ersparen. Leibnitz soll, nächst einem Aufwande von mehr als 24000 Thalern, viele seiner Nebenstunden mehrere Jahre lang dem Nachsinnen über diese Erfindung aufgeopfert haben. Der Pfarrer Hahn hat über seine Maschine 7 Jahre gearbeitet; Herr Stern in Warschau 8 Jahre, mit einem Aufwande von mehr als 10000 Thlr.; und 
neulich hat das Riesenwerk von Babbage in England 6 Jahre Zeit und einen Kosten-Aufwand von $17000 \mathrm{Pfd}$. St. erfordert. Dergleichen künstliche Werkzeuge oder sogenannte Rechenmaschinen sind in der That zu verschiedenen Zeiten zu Tage gebracht worden; und mehreren glaubten ihre Erfinder einen so sichern Mechanismus und so viel Einfachheit gegeben zu haben, dafs sie sie für den allgemeinen Gebrauch passend hielten. Schon Müller soll für die von ihm im Jahr 1784 erfundene Rechenmaschine eine gröfsere Verbreitung gesucht haben. Allein bei aller Sicherheit und allen Vortheilen, die er bei derselben nachwies, gelang es ihm doch nicht, Theilnahme dafür zu gewinnen. Auch mehrere Gelehrte, die nach ihm die Rechenmaschinen vervollkommnet haben, versuchten vergebens, denselben allgemeinen Eingang zu verschaffen.

Die Ursach dieser Fofahrung, welche ich bei der von mir vor einigen Jahren erfundenen Maschine genauer erkannt habc, liegt nicht etwa in der Unvollkommenheit der Werkzeuge, sondern darin, dafs es schwer ist, den Rechnenden zu vermögen, seine Aufmerksamkeit, die er auf die gewöhnlichen Rechen-Operationen wenden mufs, in die behutsame Aufmerksamkeit auf die Manipulation einer Maschine zu verwandeln, und dann die Sicherheit beim Rechnen, ohne eigene Überzeugung, einem unsichtbaren, der Beschädigung ausgesetzlen Räderwerke anzuvertrauen. In der That ist immer eine Verletzung oder Stockung bei einer Maschine zu besorgen, deren meisten Theile innerlich in ununterbrochener Bewegung getrieben werden, und die bald in bestimmten Puncten genau in einander greifen, bald wieder aufser Berührung kommen. Auch ist nicht, wie z. B. bei einer Uhr, die innere Beschädigung sogleich äufserlich sichtbar; vielmehr kann, da die Maschine selbst still steht, durch Bewegung der Kurbel sowohl ein falsches, als ein richtiges Resultat hervorgebracht werden.

Auch haben die mechanischen Rechenmaschinen noch den Mangel, dafs, wenn das Resultat erlangt ist, die Operation nicht mehr übersehen werden kann, um Überzeugung von deren Richtigkeit zu haben; wogegen man bei den gewöhnlichen Operationen mit der Feder noch immer wieder untersuchen kann, ob und wo ein Fehler sich eingeschlichen habe, den man dann sogleich zu verbessern im Stande ist. Mit der Maschine mufs man eines aus Unvorsichtigkeit begangenen Fehlers wegen die ganze Manipulation wiederholen. Besonders ist ein Fehler bei der Division leicht; so dafs sich behaupten läfst, es habe bis jetzt noch keine wirkliche Divisionsmaschine, selbst auf dem mechanischen Wege, gegeben. Denn da bei diesen Maschinen die Division in eine 
Subtraction verwandelt werden soll, so mufs der Theil der Maschine, auf welcher der Divisor steht, unter die gehörigen Ziffern des Dividendus gebracht und von denselben so oftmal abgedreht werden, als die Subtraction möglich ist; und dann mufs wieder der bewegliche Theil um eine Stelle weiter gerückt und auf die nemliche Weise für jede Ziffer des Quotienten verfahren werden. Der Rechner mufs also bei jeder Umdrehung der Kurbel genau beobachten, ob der Dividendus nicht schon kleiner als der Divisor geworden ist. Hat er durch Versehen die Kurbel sogleich zu hemmen verfehlt, so ist seine ganze bisherige Rechnung vergeblich, und die Operation mufs von neuem gemacht werden. Bei meiner mechanischen Rechenmaschine ist dies schon der Fall nicht; weil die Kurbel, sammt dem Räderwerk, sowohl rechts, bei der Addition und Multiplication, als bei der Subtraction und Division links gedreht werden kann, und also bei jedem begangenen Fehler die ersten Ziffern zurückgebracht werden können; welches besonders für die Division, für die Regel de tri und andere zusammenhängende Rechnungen von Nutzen ist.

Es ist auf solche Weise leicht zu sehen, weshalb mechanische RechenInaschinen keinen Eingang finden. Abgesehen von ihrer grofsen Kostspieligkeit und der nöthigen vorsichtigen Aufmerksamkeit auf ihre Handhabung, damit weder die Maschine, noch die Rechnung leide, wird man auch bei einem für zuverlässig gehaltenen Iustrumente Rechnungen, die man oft dem Kopfrechner nicht zutraute, nicht einem Mechanismus anvertrauen wollen, von dessen innerem guten Zustande und von dessen richtigem Gebrauch, ja selbst, ob beim Anfange der Rechnung keine fremde Zahl in der Maschine gewesen sei, man sich nur vermittels des gewöhnlichen Nachrechnens zu überzeugen im Stande ist.

Man hat sich deshalb auch um tabellarische Hülfsmittel bemüht; das. heifst, um Mittel, die ihren Grund in bereits fertig ausgerechneten Zahlen haben, und durch welche gewissermafsen vermittels der vor unsern Augen geschehenden Operationen das verlangte Resultat gefunden wird. Solche Tafeln sind zwar in Hinsicht ihrer Einfachheit zuverlässiger als die mechanischen Rechenmaschinen, aber mit allen bisherigen Versuchen dieser Art ist man noch nicht dahin gekommen, dem Rechner dadurch eine wesentliche Erleichterung zu verschaffen. Die einzige und vorzüglichste Erleichterung für Multiplicationen und Divisionen würde sein, wenn man im Stande wäre, vermittels irgend einer sichern und verläfslichen Operation von jeder vielziffrigen Zahl die $2,3,4,5,6,7,8$ und 9fachen gleich mit einemmale zu erhalten. Dann hätte man für eine Multiplication nur eine Addition und für ęine Division nur eine Subtraction zu macben. Verlangte 
man z. B. die Zahl 73948 mit 8967 zu multipliciren, šo würde man, da die 8 einfachen Producte der Zahl, nemlich das 2, 3, 4, 5, 6, 7, 8 und 9fache von 73948 schon ausgerechnet sind, von denselben nur die Producte mit $7,6,9$ und 8 unter einander, jedes um eine Stelle links gerückt, abzuschreiben und zu addiren haben; welche Summe dann das verlangte Product geben würde. Bei der Division wäre umgekehrt zu verfahren; nemlich, wenn man immer von den 8 einfachen Producten des Divisors dasjenige, welches das nächste zu den letzten Ziffern des Dividendus ist, von demselben subtrahirte, so bekäme man nach und nach alle Ziffern des Quotienten. Man ersparte also so bei der Multiplication das Multipliciren, bei der Division aber das probirte Dividiren, und nachher bei jeder gefundenen Ziffer des Quotienten das Mullipiciren.

Aber diesen Zweck auf dem sichersten Wege zu erreichen, nemlich von jeder vielziffrigen Zahl ihre 8 einfachen Producte unmiltelbar mit einemmale darzustellen, ist durch alle bisherigen Versuche noch nicht gelungen. Die bekannten Neperschen Stäbe, oder die aus denselben zusammengesetzlen Maschinen von Cuspar Wolf, geben nicht die 8 einfachen Producte jeder beliebigen Zahl vollständig, sondern man mufs noch bei jedem einzelnen Producte die Zehner jeder multiplicirten Ziffer zu den nachfolgenden Einern in Gedanken addiren; und die Zehner und Einer stehen überdies in einer ungeschickten Stellung; nemlich in einer Reihe nach einander vermengt, welches beim Addiren genaue Aufmerksamkeit erfordert und für die Multiplication unbequem und dadurch unsicher, zur Division aber gar nicht anwendbar ist, weil sich nicht die Reihe der Zahlen erkennen läfst, welche nach ihrer Zusammen-Addirung der Zehner mit den nachfolgenden Einern die nächste zum Dividendus ist.

Selbst die bekannten' Multiplicationstabellen, welche, bis zu einer gewissen Zahl, von jeder Zahl ihrē 8 einfachen Producte unmittellbar anzugeben bestimm sind, konnten den verlangten $Z$ weck nicht erfüllen. Abgesehen von der Unsicherheit derselben, da für die Correctur von Bänden starken Zahlentafeln keine Bürgschaft Statt findet, geben sie auch nicht von jeder vielziffrigen Zahl die verlangten 8 Producte unmittelbar, sondern man mufs die Ziffern jedes verlangten Products von verschiedenen Stellen her zusammenlesen und nach einer gewissen Ordnung abschreiben; welches bei der Multiplication Geduld und Vorsicht verlangt, hei der Division aber, weil sich nicht alle 8 Producte des Divisors mit einem Blicke übersehen lassen, um unter ihnen das nächste am Dividendus herauszufinden, unanwendbar ist. Sollen dagegen solche Tabellen bis zu einer 7ziffrigen Zahl vollständig sein, so dafs sie von jeder Zahl alle 8 einfachen 
Producte ohne weiteres geben, so erfordert dieses, wie Herr Crelle zu seinen Tafeln bemerkt hat, 117. Quartbände, jeden von 127 Bogen stark.

Dieses Alles erwogen, erlaube ich mir, ein neues, von mir erfundenes Rechen-Instrument, welches an Einfachheit, Sicherheit und Zuverlässigkeit vor den oben gedachten Maschinen sich auszeichnen dürfte, hiermit den Sachkennern und den Rechnern zu empfehlen.

Dasselbe besteht aus einem hölzernen Kästchen von 14 Zoll lang, 10 Zoll breit und $2 \frac{1}{4}$ Zoll hoch, welches von jeder, bis auf 7 Stellen beliebigen Zahl, die auf dem Kästchen mittels einer leichten Operation aufgestellt wird, alle 8 einfachen Producte, nämlich das $2,3,4,5,6,7,8$ und 9 fache, unmittelbar in 8 untereinander geordneten Reihen vollständig giebt, so dafs für jede Multiplication nur eine Addition und für jede Division nur eine Subtraction zu machen bleibt.

Mittels einer andern einfachen Operation mit diesem Instrumente läfst sich auch jede beliebige Quadratwurzel ausziehen. Die Wurzel der 2 ersten Ziffern jedes Quadrats giebt das Instrument unmittelbar. Stellt man das 2fache der gefundenen Wurzel auf, sie sei z. B. gleich $a$, so giebt das Instrument die 2 übrigen Glieder, nemlich $2 a b+b^{2}$ für jeden Werth von $b$, von 1 bis 9 . Man darf dann nur das dieser Columne des Quadrats nächste Product von derselben subtrahiren und mit dem gebliebenen Rest auf die nämliche Weise u.s. w. verfahren, so findet man nach einander alle Ziffern der Wurzel. Es werden also so die beschwerlichsten Operationen bei jeder Ausziehung der Quadratwurzel, nemlich die Versuche, um die richtige Wurzel zu treffen und nachher mit derslben das Glied $2 a+b$ zu multipliciren, erspart.

Die Vorzüge des Instruments vor den bis jetzt bekannten Hülfsmitleln sind einleuchtend. Bei den gewöhnlichen mechanischen Rechenmaschinen, wenn sie auch alle Additionen und Subtracionen verrichten, wiegen die Vortheile die vielen oben bemerkten Nachtheile nicht auf; die sie vielmehr zum allgemeinen Gebrauch ungeschickt machen. Dagegen hat das Instrument vor den mechunischen Mitteln folgende Vorzüge.

1. Es ist zucerlïssiy, weil es seine Resultate nicht auf mechanischem Wege hervorbringt, nemlich nicht durch Bewegung. Es beruht vielmehr auf einem besondern Zahlentheorem. Ich habe dieses Theorem Herrn Crelle mitgetheilt, und er hat einen allgemeinen Beweis davon gefunden. Der kleine Mechanismus des Instruments nimmt an den Berechnungen gar keinen Antheil, sondern ist blofs da, um die für die Anwendung des Theorems erforderliche 
Manipulation schnell und leicht hervorzubringen. Ist dieselbe hervorgebracht, so liegt die Richtigkeit des Resultats dem Rechner deutlich vor Augen, ohne weiter dem Mechanismus im geringsten vertrauen zu dürfen, während man bei den mechanischen Rechenmaschinen sich blindlings auf verborgene und complicirte Mechanismen verlassen mufs.

2. Bei jedem Resultat bleibt sowohl die erste aufgestellte Zahl, als die ganze Operation noch sichtbar, so dafs der Rechner mit einem Überblick von der Richtigkeit des Geschehenen sich überzeugen kann; auch wenn er die Operation durch Jemand Andern hat verrichten lassen. Hat irgend ein Fehler bei der Operation Statt gefunden, so wird er deutlich bemerkt und ist leicht abzustellen; während bei den mechanischen Rechenmaschinen, weil die Operationen selbst ganz verschwinden, ein begangener Fehler nicht zu bemerken und, wenn man ihn auch kennte, nicht mehr zu-verbessern ist.

3. Die Maschine bietet ihrer Construction nach, denn sie ist aus gleichen, massiven, mehrentheils hölzernen Theilen zusammengesetzt, deren Zusammenstellung und Bewegung höchst einfach ist, die gröfste Sicherheit dar. Sie kann fast nie Schaden leiden; und wenn es auf irgend eine Weise geschehen sollte, so ist der Schaden äufserlich sichtbar und leicht wegzuschaffen.

4. Will man vermittels dieser Maschine mit Multiplicatoren, Divisoren und Wurzeln rechnen, die mehr als 7 Stellen haben, so braucht man nur zwei Exemplare der Maschine neben einander zu setzen; diese beiden geben dann miltels einer kleinen Operation, gleich einer einzelnen Maschine, Zahlen von 12 Stellen. Überhaupt läfst sich durch $n$ nebeneinander gestellte 7ziffrige Maschinen eine Maschine für Zahlen von $6 n+1$ Ziffern herstellen.

5. Da die Maschine sehr einfach ist, so läfst sie sich von gewöhnlichen Arbeitern verfertigen und ist verhältnifsmäfsig sehr wohlfeil; sie kann, fabrikmäfsig verfertigt, höchstens 6 bis 7 Thlr. kosten.

Eine solche Maschine habe ich während meines kurzen Aufenthalts in Berlin verfertigen lassen und auch der Akademie der Wissenschaften in ihrer Sitzung vom 8ten August vorgezeigt.

Bemerkuny des Herausgebers dieses Journals. Der Herr Verfasser der vorstehenden Abhandlung erwähnt in derselben, ich habe bei meinen Rechentafeln geäufsert, die 2, 3, 4, 5, 6, 7, 8 und 9fachen aller 7ziffrigen Zahlen vollständig zu drucken, würden 117 Quartbände jeder von 127 Bogen nöthig 
sein. Dem ist allerdings so: aber ich habe auch durch die That, nemlich durch eben jene Tafeln, bewiesen, dafs sich die genannten Producte auch in einen einzigen Band von 125 Bogen bringen lassen. Die Producte stehen hier freilich nicht jedes in einer und derselben Zeile, aber sie sind recht gut eines neben dem andern zu übersehen. Auch meine frühern, in Octav gedruckten Rechentafeln vom Jahr 1820, welche die 2, 3, 4 bis 1000 fachen der Zahlen von 1 bis 1000 angeben, würden nur einen Quartband füllen. Gegen die Rechentafeln, als Hülfsmittel beim Rechnen, dürfte also wohl der Einwand, dafs sie zu voluminös sind, nicht Statt finden. Auch dafs bei dem Druck Fehler entstehen können, dürfte kein Einwand sein: denn die Druckfehler finden sich, wenn die Correctur, wie es geschehen mufs, rechnend gemacht wird, fast ohne Ausnahme; und die dennoch etwa bleibenden wenigen Fehler finden sich beim Gebrauch der Tafeln allmälig; und sind sie alle gefunden, und werden dann die Tafeln stereotypirt, so sind diese für immer fehlerfrei.

Ich will es indessen gern zugeben, dafs eine gut ausgedachte, und besonders eine recht einfache Rechenmaschine, vielen Rechnern angenehmer sein wird, als irgend eine gedruckte Rechentafel. Eine solche gute Rechenmaschine scheint mir, von den beiden, vom Herrn Verfasser aufgestellten, besonders die zweite, hier oben beschriebene zu sein. Herr Slonimsky hat die Güte gehabt, mir seine beiden Maschinen und ihren Gebrauch zu zeigen. Beide sind nach meiner Meinung ungemein sinnreich, und die zweite ist höchst einfach.

Das Zahlentheorem, auf welchem, wie der Herr Verfasser in der Abhandlung bemerkt, die zweite Maschine beruht, ist ebenfalls recht interessant; ich werde es, nebst dem Beweise desselben, auf welchen ich gekommen bin, bekannt machen, sobald der Herr Verfasser über seine Maschine verfügt haben wird. 